\title{
Synthesis, Spectroscopic Studies and Biological Applications of Organotin(IV) Derivatives of 3-[N-(4-Nitrophenyl)-amido]propenoic Acid and 3-[N-(4-Nitrophenyl)-amido]propanoic Acid
}

\author{
Khadija Shahid. Saira Shahzadi. Saqib Ali," and M. Mazhar \\ Department of Chemistry, Quaid-i-Azam University, Islamabad 45320, Pakistan. "E-mail:drsa54@yahoo.com \\ Received Jine 22, 2005
}

\begin{abstract}
New organotin(IV) derivatives with general formulae $\mathrm{R}_{2} \mathrm{SnL}_{2}$ and $\mathrm{R}_{3} \mathrm{SnL}$, where $\mathrm{R}=$ methyl, n-butyl, $\mathrm{n}$-octyl and phenyl and $\mathrm{HL}$ is either 3-[N-(4-nitrophenyl)amido]-propenoic acid or 3-[N-(4-nitrophenyl)amido] propanoic acid have been synthesized in $1: 2$ and $1: 1$ molar ratio by different methods. The FTIR spectra clearly demonstrated that the organotin(IV) moieties react with $[\mathrm{O}, \mathrm{O}]$ atoms of the ligands. The bonding and coordination behavior in these complexes are discussed on the basis of multinuclear $\left({ }^{1} \mathrm{H},{ }^{13} \mathrm{C}\right.$. $\left.{ }^{19} \mathrm{Sn}\right) \mathrm{NMR}$ and mass spectrometric studies. Antibacterial, and antifungal screening tests were performed for these compounds and reported here. These values were compared to those of the precursors and it was found that diorganotin(IV) complexes exhibit less activity as compared to triorganotin(IV) complexes. $\mathrm{LD}_{50}$ data were obtained by Brine Shrimp assay method. Insecticidal activity was performed for selective compounds by contact toxicity method.
\end{abstract}

Key Words : Organotin(IV) carboxylates, Spectroscopic characterization, Biological activity

\section{Introduction}

The synthesis of organotin(IV) carboxylates has received considerable attention during the last three decades..$^{1-10}$ Increasing interest in generating these complexes lies in the possibility to enhance biological activities associated with the respective organotin(IV) and unreacted carboxylate compounds.

The biological importance of organotins has been supported by studies concentrating on structure-activity correlations ${ }^{11-18}$ that dealt mainly with structural aspects and antitumour activity, and also linked with possible tumorigenic activity. Indeed, butyltins present genotoxic effects, ${ }^{1920}$ and may predispose animals to malignancy. Several studies have reported that ligands containing oxygen and nitrogen atoms as donor sites are often involved in compounds with potential antitumour activity. ${ }^{21-27}$

In order to obtain better insight into how the metallic species behave inside biological systems, it is necessary to study their coordination behaviour with ligands that can occur in the biological medium, and hence to formulate the structure-activity correlations to devise new derivatives with potential antitumour activity. This explains why attention has shifted towards metal derivatives of amino acids and peptides.

Our current interest in the synthesis of organotin(IV) carboxylates $^{28-32}$ prompted us to extend our investigation in the synthesis of new organotin(IV) carboxylates, which could have biological properties. This paper describes the synthesis of new organotin(IV) carboxylates of 3-[N-(4nitrophenyl)amido]propenoic acid and 3-[N-(4-nitrophenyl)amido]propanoic acid.

All compounds were characterized by IR, ${ }^{1} \mathrm{H},{ }^{13} \mathrm{C},{ }^{119} \mathrm{Sn}$ NMR and mass spectrometry. These complexes were screened against various bacteria, fungi and insects to investigate their possible as antibacterial and antifungal agents. $L_{50}$ values were obtained for these compounds by using Brine Shrimp assay method and the data are reported in this paper.

\section{Experimental Section}

Materials and Instrumentation. All the glass apparatus with standard quick fit joints was used throughout the work after cleaning and drying at $120^{\circ} \mathrm{C}$. Maleic anhydride, succinic anhydride, $p$-nitroaniline, chloroform and petroleum ether from Aldrich, glacial acetic acid from Merck and toluene from Fluka were purchased. Solvents were purified by standard methods ${ }^{33}$ while other chemicals were used as supplied. Melting points were determined with a Mitamura Rikero Kogyo (Japan) and are uncorrected. Mass spectra were recorded on a MAT 8500 Finngin (Germany). The ${ }^{1} \mathrm{H}$ and ${ }^{13} \mathrm{C}$ NMR spectra were recorded on a Brucker AM-250 $\mathrm{MHz}$ spectrometer using $\mathrm{CDCl}_{3}$ as internal reference. ${ }^{119} \mathrm{Sn}$ NMR spectra were obtained on a Brucker 250 ARX instrument with $\mathrm{Me}_{4} \mathrm{Sn}$ as external reference.

Preparation of 3-[N-(4-Nitrophenyl)-amido]propenoic Acid (HL'). A solution of maleic anhydride $(10 \mathrm{~g}, 0.1 \mathrm{~mol})$ in acetic acid $(300 \mathrm{~mL})$ was added to a solution of $p$ nitroaniline $(13.8 \mathrm{~g}, 0.1 \mathrm{~mol})$ in acetic acid $(150 \mathrm{~mL})$ and the mixture was stirred at room temperature overnight. The light yellow precipitates were filtered, washed with cold distilled $\mathrm{H}_{2} \mathrm{O}(200 \mathrm{~mL})$ and air dried. Yield $85 \%$, m.p. ${ }^{\circ} \mathrm{C}$. Analysis: Calculated for $\mathrm{C}_{10} \mathrm{H}_{8} \mathrm{~N}_{2} \mathrm{O}_{5}: \mathrm{C}, 50.85 ; \mathrm{H}, 3.41 ; \mathrm{N}, 11.86$. Found: $\mathrm{C}, 50.80 ; \mathrm{H}, 3.45 ; \mathrm{N}, 11.80 .{ }^{1} \mathrm{H} \mathrm{NMR}\left(\mathrm{CDCl}_{3}, \mathrm{ppm}\right)$, 8.03-8.06 $\delta(9.17) ; 8.14-8.18 \mathrm{~d}$ (9.17) $(-\mathrm{CH}=\mathrm{CH}-), 7.64$ $7.68 \mathrm{~m}\left(\mathrm{NO}_{2}-\mathrm{C}_{6} \mathrm{H}_{4}\right), 6.9 \mathrm{~s}(-\mathrm{NH}),{ }^{13} \mathrm{C} \mathrm{NMR}\left(\mathrm{CDCl}_{3}, \mathrm{ppm}\right)$ 137.8 (C-1), 132.3 (C-2/6), 134.6 (C-3/5), 126.3 (C-4), 
166.0 (C-7), 124.4 (C-8), 114.1 (C-9), 196.6 (C-10).

3-[N-(4-Nitrophenyl)-amido]propanoic Acid $\left(\mathrm{HL}^{2}\right)$. A solution of succinic anhydride $(9.8 \mathrm{~g}, 0.1 \mathrm{~mol})$ in acetic acid $(300 \mathrm{~mL})$ was added to a solution of $p$-nitroaniline $(13.80 \mathrm{~g}$, $0.099 \mathrm{~mol})$ in acetic acid $(150 \mathrm{~mL})$ and the mixture was stirred at room temperature overnight. The light yellow precipitates were filtered, washed with cold distilled $\mathrm{H}_{2} \mathrm{O}$ $(200 \mathrm{~mL})$ and air dried. Yield $85 \%$, m.p. ${ }^{\circ} \mathrm{C}$. Analysis: Calculated for $\mathrm{C}_{10} \mathrm{H}_{10} \mathrm{~N}_{2} \mathrm{O}_{5}: \mathrm{C}, 50.42 ; \mathrm{H}, 4.23 ; \mathrm{N}, 11.76$. Found: C, $50.45 ; \mathrm{H}, 4.19 ; \mathrm{N}, 11.71$. ' $\mathrm{H} \mathrm{NMR}\left(\mathrm{CDCl}_{3}, \mathrm{ppm}\right)$, $8.07-8.11 \mathrm{~d}(9.2) ; 8.34-8.36 \mathrm{~d}(9.2)\left(-\mathrm{CH}_{2}-\mathrm{CH}_{2}-\right), 7.60-7.63 \mathrm{~m}$ $\left(\mathrm{NO}_{2}-\mathrm{C}_{4} \mathrm{H}_{4}\right), 7.28 \mathrm{~s}(-\mathrm{NH}) .{ }^{13} \mathrm{C}$ NMR $\left(\mathrm{CDCl}_{3}, \mathrm{ppm}\right), 136.9$ (C-1), 132.0 (C-2/6), 134.4 (C-3/5), 126.8 (C-4), 167.0 (C7), 124.3 (C-8), 118.2 (C-9), 192.1 (C-10).

Preparation of Complexes $\mathrm{Me}_{2} \mathrm{Sn}\left(\mathrm{L}^{\prime}\right)_{2}$ (1): $3-[\mathrm{N}-(4-$ Nitrophenyl)amido]propenoic acid ( $1 \mathrm{~g}, 4.23 \mathrm{mmol}$ ) was suspended in dry toluene $(100 \mathrm{~mL})$ and treated with triethylamine $(0.59 \mathrm{~mL}, 4.23 \mathrm{mmol})$. The mixture was refluxed for $2-3$ hours. To a solution of triethylammonium 3-[N-(4nitrophenyl)amido]propenoate in dry toluene, dimethyltin dichloride $(2.11 \mathrm{mmol})$ was added as a solid to a reaction flask with constant stirring and reaction mixture was refluxed for 8-10 hours. The reaction mixture contained $\mathrm{Et}_{3} \mathrm{NHCl}$ was filtered off such that filtrate had the organotin(IV) derivative. The solvent was removed through rotary apparatus under reduced pressure. The mass left behind was recrystallized from $\mathrm{CHCl}_{3}$ and pet.ether $(1: 1)$. Yield $85 \%$, m.p. $105{ }^{\circ} \mathrm{C}$. Analysis: Calculated for $\mathrm{C}_{20} \mathrm{H}_{20} \mathrm{~N}_{4} \mathrm{O}_{10} \mathrm{Sn}$ : C, $38.77 ; \mathrm{H}, 3.23 ; \mathrm{N}, 9.04$. Found: C, 38.65; $\mathrm{H}, 3.20 ; \mathrm{N}, 9.12$. 'H NMR ( $\left.\mathrm{CDCl}_{3}, \mathrm{ppm}\right), 8.02-8.06 \mathrm{~d}(8.98)$; $8.14-8.32 \mathrm{~d}(8.98)(-\mathrm{CH}=\mathrm{CH}-), 7.63-7.67 \mathrm{~m}\left(\mathrm{NO}_{2}-\mathrm{C}_{4} \mathrm{H}_{4}\right), 6.9 \mathrm{~s}$ $(-\mathrm{NH}), 1.2 \mathrm{~s}[78.5]\left(\mathrm{Sn}_{11}-\mathrm{CH}_{3}\right) .{ }^{13} \mathrm{C} \mathrm{NMR}\left(\mathrm{CDCl}_{3}, \mathrm{ppm}\right), 138.0$ (C-1), 132.6 (C-2/6), 134.6 (C-3/5), 126.3 (C-4), 168.4 (C7), 124.4 (C-8), 113.3 (C-9), 178.4 (C-10), 29.68 (C-11).

IR $\left(\mathrm{KBr}, \mathrm{cm}^{-1}\right), 3392 \mathrm{~s} v(\mathrm{NH}), 1720 \mathrm{~s} v(\mathrm{C}=\mathrm{O}), 1555 \mathrm{~s} v$ $\left(\mathrm{COO}_{2 s y \mathrm{~m}}\right), 1402 \mathrm{~s} v\left(\mathrm{COO}_{\mathrm{sym}}\right), \Delta v 155,580 \mathrm{~m} v(\mathrm{Sn}-\mathrm{C})$, $405 \mathrm{~m} v$ (Sn-O). Mass data $\mathrm{m} / \mathrm{z}(\%)$; RSnOOCR' 368 (n.o), SnOOCR' 353 (n.o), $\mathrm{R}_{2} \mathrm{Sn}^{+} 149$ (n.o), $\mathrm{C}_{6} \mathrm{H}_{5}{ }^{+} 76$ (8), [Sn] ${ }^{+}$ $120(22), \mathrm{C}_{7} \mathrm{H}_{5} \mathrm{O}_{3} \mathrm{~N}^{+} 165(6), \mathrm{CNHO}^{+} 43(6),\left[\mathrm{C}_{6} \mathrm{H}_{5}\right]^{+} 77(2)$, $\mathrm{C}_{6} \mathrm{H}_{5} \mathrm{~N}_{2} \mathrm{O}_{2}{ }^{+} 138(100) . \delta\left({ }^{119} \mathrm{Sn}\right) \mathrm{ppm} ;-98.3$.

$\mathbf{P h}_{2} \mathbf{S n}\left(\mathbf{L}^{\prime}\right)_{2}$ (2): 3-[N-(4-Nitrophenyl)amido]propenoic acid $(1 \mathrm{~g}, 4.23 \mathrm{mmol})$ was suspended in dry toluene $(100$ $\mathrm{mL})$ and treated with triethylamine $(0.59 \mathrm{~mL}, 4.23 \mathrm{mmol})$. The mixture was refluxed for $2-3$ hours. To a solution of triethylammonium 3-[N-(4-nitrophenyl)amido]propenoate in dry toluene, diphenyltin dichloride $(2.11 \mathrm{mmol})$ was added as a solid to a reaction flask with constant stirring and reaction mixture was refluxed for $8-10$ hours. The reaction mixture contained $\mathrm{Et}_{3} \mathrm{NHCl}$ was filtered off such that filtrate had the organotin(IV) derivative. The solvent was removed through rotary apparatus under reduced pressure. The mass left behind was recrystallized from $\mathrm{CHCl}_{3}$ and pet.ether $(1$ : 1). Yield $70 \%$, m.p. $121-122{ }^{\circ} \mathrm{C}$. Analysis: Calculated for $\mathrm{C}_{32} \mathrm{H}_{24} \mathrm{~N}_{4} \mathrm{O}_{10} \mathrm{Sn}: \mathrm{C}, 51.68 ; \mathrm{H}, 3.23 ; \mathrm{N}, 7.53$. Found: C, 51.32; $\mathrm{H}, 3.30 ; \mathrm{N}, 7.49$.

${ }^{1} \mathrm{H}$ NMR ( $\left.\mathrm{CDCl}_{3}, \mathrm{ppm}\right), 8.05-8.06 \mathrm{~d}(9.08) ; 8.35-8.36 \mathrm{~d}$ (9.08) $(-\mathrm{CH}=\mathrm{CH}-), 7.68-7.71 \mathrm{~m}\left(\mathrm{NO}_{2}-\mathrm{C}_{6} \mathrm{H}_{4}\right), 6.9 \mathrm{~s}(-\mathrm{NH})$,
$7.28 \mathrm{~m}\left(\mathrm{Sn}_{-}-\mathrm{C}_{6} \mathrm{H}_{5}\right) .{ }^{13} \mathrm{C}$ NMR $\left(\mathrm{CDCl}_{3}, \mathrm{ppm}\right), 137.1(\mathrm{C}-1)$, 132.4 (C-2/6), 134.6 (C-3/5), 126.7 (C-4), 168.5 (C-7), 124.4 (C-8), 113.3 (C-9), 176.8 (C-10), 137.1 (C-11), 136.1 (C-12), 129.1 (C-13), 128.4 (C-14).

IR $\left(\mathrm{KBr}, \mathrm{cm}^{-1}\right), 3378 \mathrm{~s} v(\mathrm{NH}), 1725 \mathrm{~s} v(\mathrm{C}=\mathrm{O}), 1599 \mathrm{~s} v$ $\left(\mathrm{COO}_{\text {as.m }}\right), 1424 \mathrm{~s} v\left(\mathrm{COO}_{\mathrm{sym}}\right), \Delta v 175,440 \mathrm{~s} v(\mathrm{Sn}-\mathrm{O})$ ). Mass data $\mathrm{m} / \mathrm{z}(\%)$; RSnOOCR' 429 (5), SnOOCR' $353(4), \mathrm{R}_{2} \mathrm{Sn}^{+}$ 271 (7), $\mathrm{C}_{6} \mathrm{H}_{5}^{+} 76$ (n.o), [Sn] $120(2), \mathrm{C}_{7} \mathrm{H}_{5} \mathrm{O}_{3} \mathrm{~N}^{+} 165$ (2), $\mathrm{CNHO}^{+} 43(9),\left[\mathrm{C}_{6} \mathrm{H}_{5}\right]^{+} 77(6), \mathrm{C}_{6} \mathrm{H}_{5} \mathrm{~N}_{2} \mathrm{O}_{2}{ }^{+} 138(100) . \delta$ ("195n) ppm; -98.4.

$\operatorname{Oct}_{2} \operatorname{Sn}\left(\mathbf{L}^{1}\right)_{2} \quad$ (3): 3-[N-(4-Nitrophenyl)amido]propenoic acid $(1 \mathrm{~g}, 4.23 \mathrm{mmol}$ ) was suspended in dry toluene (100 $\mathrm{mL}$ ) and treated with equimolar in a reaction flask with constant stirring and mixture was refluxed for 8-10 hours. Water formed was removed via Dean and Stark trap. The solvent was removed through rotary apparatus under reduced pressure. The mass left behind was recrystallized from $\mathrm{CHCl}_{3}$ and pet.ether $(1: 1)$. Yield $86 \%$, m.p. $98^{\circ} \mathrm{C}$. Analysis: Calculated for $\mathrm{C}_{36} \mathrm{H}_{48} \mathrm{~N}_{4} \mathrm{O}_{10} \mathrm{Sn}: \mathrm{C}, 53.00 ; \mathrm{H}, 5.88$; $\mathrm{N}, 6.87$. Found: C, $53.20 ; \mathrm{H}, 5.77 ; \mathrm{N}, 6.84$.

'H NMR ( $\left.\mathrm{CDCl}_{3}, \mathrm{ppm}\right), 8.02-8.05 \mathrm{~d}(9.08)$ 8.32-8.36d (9.08) $(-\mathrm{CH}=\mathrm{CH}-), 7.60-7.68 \mathrm{~m}\left(\mathrm{NO}_{2}-\mathrm{C}_{6} \mathrm{H}_{4}\right), 6.9 \mathrm{~s}(-\mathrm{NH})$, $0.83-1.9 \mathrm{~m}\left(\mathrm{Sn}_{-} \mathrm{C}_{8} \mathrm{H}_{13}\right) .{ }^{13} \mathrm{C} \mathrm{NMR}\left(\mathrm{CDCl}_{3}, \mathrm{ppm}\right), 138.2(\mathrm{C}-$ 1), 132.5 (C-2/6), 134.6 (C-3/5), 126.3 (C-4), 168.4 (C-7), 124.4 (C-8), 114.5 (C-9), 175.0 (C-10), 34.1 (C-11), 31.9 (C12), 29.5 (C-13), 26.3 (C-14), 26.3 (C-15), 25.8 (C-16), 23.4 (C-17), $14.9(\mathrm{C}-18)$.

IR $\left(\mathrm{KBr}, \mathrm{cm}^{-1}\right), 3349 \mathrm{~s} v(\mathrm{NH}), 1701 \mathrm{~m} v(\mathrm{C}=\mathrm{O}), 1560 \mathrm{~s} v$ $\left(\mathrm{COO}_{\text {as:m }}\right), 1412 \mathrm{~s} v\left(\mathrm{COO}_{\mathrm{s} \mathrm{m}}\right), \Delta v 148,520 \mathrm{~m} v(\mathrm{Sn}-\mathrm{C})$, $410 \mathrm{~m} v$ (Sn-O). Mass data $\mathrm{m} / \mathrm{z}(\%)$; RSnOOCR 465 (n.o), SnOOCR' 353 (2), $\mathrm{R}_{2} \mathrm{Sn}^{+} 345$ (10), $\mathrm{C}_{6} \mathrm{H}_{5}{ }^{+} 76$ (n.o), [Sn] 120 (n.o), $\mathrm{C}_{7} \mathrm{H}_{5} \mathrm{O}_{3} \mathrm{~N}^{+} 165$ (n.o), $\mathrm{CNHO}^{+} 43$ (12), $\left[\mathrm{C}_{6} \mathrm{H}_{5}\right]^{+} 77$ (n.o), $\mathrm{C}_{6} \mathrm{H}_{5} \mathrm{~N}_{2} \mathrm{O}_{2}^{+} 138(100)$.

$\mathrm{Me}_{2} \mathrm{Sn}\left(\mathbf{L}^{2}\right)_{2}$ (4): 3-[N-(4-nitro-phenylamido]propanoic acid $(1 \mathrm{~g}, 4.20 \mathrm{mmol})$ was suspended in dry toluene $(100$ $\mathrm{mL}$ ) and treated with triethylamine $(0.58 \mathrm{~mL}, 4.20 \mathrm{mmol})$. The mixture was refluxed for 2-3 hours. To a solution of triethylammonium 3-[N-(4-nitrophenyl)amido]propanoate in dry toluene, dimethyltin dichloride $(2.10 \mathrm{mmol})$ was added as a solid to a reaction flask with constant stirring and reaction mixture was refluxed for $8-10$ hours. The reaction mixture contained $\mathrm{Et}_{3} \mathrm{NHCl}$ was filtered off such that filtrate had the organotin(IV) derivative. The solvent was removed through rotary apparatus under reduced pressure. The mass left behind was recrystallized from $\mathrm{CHCl}_{3}$ and pet.ether (1: 1). Yield $86 \%$, m.p. $92{ }^{\circ} \mathrm{C}$. Analysis: Calculated for $\mathrm{C}_{22} \mathrm{H}_{24} \mathrm{~N}_{4} \mathrm{O}_{10} \mathrm{Sn}: \mathrm{C}, 42.37 ; \mathrm{H}, 3.85 ; \mathrm{N}, 8.98$. Found: C, 41.98 ; $\mathrm{H}, 3.79 ; \mathrm{N}, 9.10$.

'H NMR ( $\mathrm{CDCl}_{3}$, ppm), 8.11-8.15d (9.0); 8.38-8.42d (9.0) $\left(-\mathrm{CH}_{2}-\mathrm{CH}_{2}-\right), 7.64-7.67 \mathrm{~m}\left(\mathrm{NO}_{2}-\mathrm{C}_{6} \mathrm{H}_{4}\right), 7.32 \mathrm{~s}(-\mathrm{NH}), 1.31 \mathrm{~s}$ [79.1] $\left(\mathrm{Sn}^{\left.-\mathrm{CH}_{3}\right)},{ }^{13} \mathrm{C} \mathrm{NMR}\left(\mathrm{CDCl}_{3}, \mathrm{ppm}\right), 137.8(\mathrm{C}-1)\right.$, 132.6 (C-2/6), 134.5 (C-3/5), 126.3 (C-4), 167.6 (C-7), 124.4 (C-8), 118.3 (C-9), 175.3 (C-10), 29.7 (C-11).

$\mathbb{R}\left(\mathrm{KBr}, \mathrm{cm}^{-1}\right), 3362 \mathrm{~s} v(\mathrm{NH}), 1752 \mathrm{~s} v(\mathrm{C}=\mathrm{O}), 1586 \mathrm{~s} v$ $\left(\mathrm{COO}_{\text {asym }}\right), 1440 \mathrm{~s} v\left(\mathrm{COO}_{\mathrm{syn}}\right), \Delta v 146,542 \mathrm{~m} v(\mathrm{Sn}-\mathrm{C})$, $452 \mathrm{~m} v($ Sn-O). Mass data $\mathrm{m} / \mathrm{z}(\%)$; RSnOOCR' 608 (n.o), SnOOCR' 593 (n.o), $\mathrm{R}_{2} \mathrm{Sn}^{+} 149$ (6), $\mathrm{C}_{6} \mathrm{H}_{5}^{+} 76(5),[\mathrm{Sn}]^{+} 120$ 
(6), $\mathrm{C}_{3} \mathrm{H}_{4} \mathrm{O}^{+} 55(68),\left[\mathrm{R}_{2} \mathrm{SnOOCCH}_{2} \mathrm{CH}_{2}\right]^{+} 220(100)$, $\mathrm{C}_{7} \mathrm{H}_{5} \mathrm{O}_{3} \mathrm{~N}^{+} 165(10), \mathrm{CNHO}^{+} 43(18), \mathrm{C}_{3} \mathrm{H}_{5} \mathrm{NO}^{+} 71(19)$, $\left[\mathrm{C}_{6} \mathrm{H}_{5}\right]^{+} 77(1), \mathrm{C}_{6} \mathrm{H}_{5} \mathrm{~N}_{2} \mathrm{O}_{2}{ }^{+} 138(5)$.

$\mathrm{Bu}_{2} \operatorname{Sn}\left(\mathbf{L}^{2}\right)_{2}$ (5): 3-[N-(4-nitro-phenylamido]propanoic acid $(1 \mathrm{~g}, 4.20 \mathrm{mmol})$ was suspended in dry toluene (100 $\mathrm{mL}$ ) and treated with triethylamine $(0.58 \mathrm{~mL}, 4.20 \mathrm{mmol})$. The mixture was refluxed for 2-3 hours. To a solution of triethylammonium 3-[N-(4-nitrophenyl)amido]propanoate in dry toluene, dibutyltin dichloride $(2.10 \mathrm{mmol})$ was added as a solid to a reaction flask with constant stirring and reaction mixture was refluxed for $8-10$ hours. The reaction mixture contained $\mathrm{Et}_{3} \mathrm{NHCl}$ was filtered off such that filtrate had the organotin(IV) derivative. The solvent was removed through rotary apparatus under reduced pressure. The mass left behind was recrystallized from $\mathrm{CHCl}_{3}$ and pet.ether $(1$ : 1). Yield $90 \%$, m.p. $62{ }^{\circ} \mathrm{C}$. Analysis: Calculated for $\mathrm{C}_{28} \mathrm{H}_{36} \mathrm{~N}_{4} \mathrm{O}_{10} \mathrm{Sn}: \mathrm{C}, 47.52 ; \mathrm{H}, 5.09 ; \mathrm{N}, 7.92$. Found: C, 47.45; $\mathrm{H}, 5.11 ; \mathrm{N}, 8.01$.

${ }^{1} \mathrm{H} \mathrm{NMR}\left(\mathrm{CDCl}_{3}, \mathrm{ppm}\right), 8.10-8.13 \mathrm{~d}(9.0) ; 8.37-8.41 \mathrm{~d}(9.0)$ $\left(-\mathrm{CH}_{2}-\mathrm{CH}_{2}-\right), 7.63-7.71 \mathrm{~m}\left(\mathrm{NO}_{2}-\mathrm{C}_{4} \mathrm{H}_{4}\right), 7.32 \mathrm{~s}(-\mathrm{NH}), 0.8 \mathrm{t}$, $1.30-1.55 \mathrm{~m}\left(\mathrm{Sn}-\mathrm{C}_{4} \mathrm{H}_{9}\right) .{ }^{13} \mathrm{C} \mathrm{NMR}\left(\mathrm{CDCl}_{3}, \mathrm{ppm}\right), 137.6(\mathrm{C}-$ 1), 132.8 (C-2/6), 134.2 (C- $3 / 5), 126.3$ (C-4), 167.8 (C-7), 124.4 (C-8), 113.3 (C-9), 175.3 (C-10), 29.7 (C-11), 27.3 (C12), 26.7 (C-13), 13.6 (C-14).

IR $\left(\mathrm{KBr} \mathrm{cm}^{-1}\right), 3351 \mathrm{~s} v(\mathrm{NH}), 1742 \mathrm{~s} v(\mathrm{C}=\mathrm{O}), 1578 \mathrm{~s} v$ $\left(\mathrm{COO}_{2 s y \mathrm{~m}}\right), 1420 \mathrm{~s} v\left(\mathrm{COO}_{\mathrm{sym}}\right), \Delta v 158,552 \mathrm{w} v(\mathrm{Sn}-\mathrm{C})$, $480 \mathrm{~m} v$ (Sn-O). Mass data m/z(\%); RSnOOCR' 650 (n.o), SnOOCR' 593 (n.o), $\mathrm{R}_{2} \mathrm{Sn}^{+} 233$ (n.o), $\mathrm{C}_{6} \mathrm{H}_{5}^{+} 76$ (n.o), [Sn] $]^{+}$ $120(8), \mathrm{C}_{3} \mathrm{H}_{4} \mathrm{O}^{+} 55$ (3), $\left[\mathrm{R}_{2} \mathrm{SnOOCCH}_{2} \mathrm{CH}_{2}\right]^{+} 220$ (8), $\mathrm{C}_{7} \mathrm{H}_{5} \mathrm{O}_{3} \mathrm{~N}^{+} 165(2), \mathrm{CNHO}^{+} 43(8), \mathrm{C}_{3} \mathrm{H}_{5} \mathrm{NO}^{+} 71(13)$, $\left[\mathrm{C}_{6} \mathrm{H}_{5}\right]^{+} 77(9), \mathrm{C}_{6} \mathrm{H}_{5} \mathrm{~N}_{2} \mathrm{O}_{2}{ }^{+} 138(9), \mathrm{C}_{4} \mathrm{H}_{9}{ }^{+} 57(100) . \delta\left({ }^{119} \mathrm{Sn}\right)$ ppm; -88.3 .

$\operatorname{Oct}_{2} \operatorname{Sn}\left(\mathbf{L}^{2}\right)_{2} \quad$ (6): 3-[N-(4-Nitrophenyl)amido]propanoic acid $(1 \mathrm{~g}, 4.20 \mathrm{mmol}$ ) was suspended in dry toluene (100) $\mathrm{mL}$ ) and treated with equimolar in a reaction flask with constant stirring and mixture was refluxed for $8-10$ hours. Water formed was removed via Dean and Stark trap. The solvent was removed through rotary apparatus under reduced pressure. The mass left behind was recrystallized from $\mathrm{CHCl}_{3}$ and pet.ether $(1: 1)$.

Yield $78 \%$, m.p. $170{ }^{\circ} \mathrm{C}$. Analysis: Calculated for $\mathrm{C}_{36} \mathrm{H}_{52} \mathrm{~N}_{4} \mathrm{O}_{10} \mathrm{Sn}: \mathrm{C}, 52.74 ; \mathrm{H}, 6.34 ; \mathrm{N}, 6.83$. Found: $\mathrm{C}, 52.63$; $\mathrm{H}, 6.39 ; \mathrm{N}, 6.81$.

${ }^{1} \mathrm{H} \mathrm{NMR}\left(\mathrm{CDCl}_{3}, \mathrm{ppm}\right), 8.07-8.10 \mathrm{~d}(9.0) ; 8.34-8.37 \mathrm{~d}(9.0)$ $\left(-\mathrm{CH}_{2}-\mathrm{CH}_{2}-\right), 7.60-7.69 \mathrm{~m}\left(\mathrm{NO}_{2}-\mathrm{C}_{6} \mathrm{H}_{4}\right), 7.28 \mathrm{~s}(-\mathrm{NH}), 0.89-$ $1.73 \mathrm{~m}\left(\mathrm{Sn}-\mathrm{C}_{6} \mathrm{H}_{5}\right) .{ }^{13} \mathrm{C}$ NMR $\left(\mathrm{CDCl}_{3}, \mathrm{ppm}\right), 137.2(\mathrm{C}-1)$, 132.4 (C-2/6), 134.9 (C-3/5), 126.9 (C-4), 167.2 (C-7), 124.4 (C-8), 118.6 (C-9), 175.8 (C-10), 33.9 (C-11), 31.9 (C12), 29.6 (C-13), 29.3 (C-14), 26.3 (C-15), 25.3 (C-16), 24.2 (C-17), $12.3(\mathrm{C}-18)$.

IR $\left(\mathrm{KBr}, \mathrm{cm}^{-1}\right)$, 3395s $v(\mathrm{NH}), 1732 \mathrm{~s} v(\mathrm{C}=\mathrm{O}), 1580 \mathrm{~s} v$ $\left(\mathrm{COO}_{\mathrm{assnn}}\right), 1400 \mathrm{~s} v\left(\mathrm{COO}_{\mathrm{sym}}\right), \Delta v 180,532 \mathrm{~m} v(\mathrm{Sn}-\mathrm{C})$, $472 \mathrm{~m} v$ (Sn-O). Mass data $\mathrm{m} / \mathrm{z}(\%)$; RSnOOCR' 706 (n.o), SnOOCR' 356 (100), $\mathrm{R}_{2} \mathrm{Sn}^{+} 345$ (n.o), $\mathrm{C}_{6} \mathrm{H}_{5}^{+} 76$ (6), [Sn] $]^{+}$ $120(5), \mathrm{C}_{3} \mathrm{H}_{4} \mathrm{O}^{+} 55(100),\left[\mathrm{R}_{2} \mathrm{SnOOCCH}_{2} \mathrm{CH}_{2}\right]^{+} 220(97)$, $\mathrm{C}_{7} \mathrm{H}_{5} \mathrm{O}_{3} \mathrm{~N}^{+} 165(16), \mathrm{CNHO}^{+} 43(11), \mathrm{C}_{3} \mathrm{H}_{5} \mathrm{NO}^{+} 71(18)$, $\left[\mathrm{C}_{6} \mathrm{H}_{5}\right]^{+} 77(6), \mathrm{C}_{6} \mathrm{H}_{5} \mathrm{~N}_{2} \mathrm{O}_{2}{ }^{+} 138(12) . \delta\left({ }^{119} \mathrm{Sn}\right) \mathrm{ppm} ;-148.7$.
$\mathbf{M e}_{3} \operatorname{Sn}\left(\mathbf{L}^{\prime}\right)$ (7): 3-[N-(4-Nitrophenyl)amido]propenoic acid $(1 \mathrm{~g}, 4.23 \mathrm{mmol})$ was suspended in dry toluene (100 $\mathrm{mL}$ ) and treated with triethylamine $(0.59 \mathrm{~mL}, 4.23 \mathrm{mmol})$. The mixture was refluxed for 2-3 hours. To a solution of triethylammonium 3-[N-(4-nitrophenyl)amido]propenoate in dry toluene, trimethyltin chloride $(4.23 \mathrm{mmol})$ was added as a solid to a reaction flask with constant stirring and reaction mixture was refluxed for $8-10$ hours. The reaction mixture contained $\mathrm{Et}_{3} \mathrm{NHCl}$ was filtered off such that filtrate had the organotin(IV) derivative. The solvent was removed through rotary apparatus under reduced pressure. The mass left behind was recrystallized from $\mathrm{CHCl}_{3}$ and pet.ether $(1$ : 1). Yield $77 \%$, m.p. $45-46{ }^{\circ} \mathrm{C}$. Analysis: Calculated for $\mathrm{C}_{13} \mathrm{H}_{17} \mathrm{~N}_{2} \mathrm{O}_{5} \mathrm{Snn}: \mathrm{C}, 39.00 ; \mathrm{H}, 4.25 ; \mathrm{N}, 7.00$. Found: C, 39.22; $\mathrm{H}, 4.19$; N, 7.11.

'H NMR ( $\left.\mathrm{CDCl}_{3}, \mathrm{ppm}\right), 8.00-8.03 \mathrm{~d}(9.08) ; 8.09-8.13 \mathrm{~d}$ (9.08) (-CH=CH-), 7.63-7.66m $\left(\mathrm{NO}_{2}-\mathrm{C}_{6} \mathrm{H}_{4}\right), 6.9 \mathrm{~s}(-\mathrm{NH})$, $0.74 \mathrm{~s}[57.17]\left(\mathrm{Sn}_{-}-\mathrm{CH}_{3}\right),{ }^{13} \mathrm{C} \mathrm{NMR}\left(\mathrm{CDCl}_{3}\right.$, ppm), $137.9(\mathrm{C}-$ 1), 132.5 (C-2/6), 134.2 (C-3/5), 126.3 (C-4), 168.5 (C-7), 124.4 (C-8), 113.3 (C-9), 181.6 (C-10), 29.68 (C-11). IR $\left(\mathrm{KBr}, \mathrm{cm}^{-1}\right), 3334 \mathrm{~s} v(\mathrm{NH}), 1724 \mathrm{~s} v(\mathrm{C}=\mathrm{O}), 1597 \mathrm{~s} v$ $\left(\mathrm{COO}_{2 s \mathrm{~m}}\right), 1415 \mathrm{~s} v\left(\mathrm{COO}_{\mathrm{ssm}}\right), \Delta v 182,550 \mathrm{~m} v(\mathrm{Sn}-\mathrm{C})$, $420 \mathrm{~m} v$ (Sn-O). Mass data $\mathrm{m} / \mathrm{z}(\%)$; R2 $\mathrm{SnOOCR}^{\prime} 385$ (43), RSnOOCR' 370 (35), SnOOCR' 354 (2), $\mathrm{R}_{3} \mathrm{Sn}^{+} 164$ (5), $\mathrm{R}_{2} \mathrm{Sn}^{+} 149(12), \mathrm{C}_{6} \mathrm{H}_{5}^{+} 76(9),[\mathrm{Sn}]^{+} 120(4), \mathrm{C}_{7} \mathrm{H}_{5} \mathrm{O}_{3} \mathrm{~N}^{+} 165$ (n.o), $\mathrm{CNHO}^{+} 43(20),\left[\mathrm{C}_{6} \mathrm{H}_{7}\right]^{+} 77$ (n.o), $\mathrm{C}_{6} \mathrm{H}_{5} \mathrm{~N}_{2} \mathrm{O}_{2}{ }^{+} 138$ (100). $\delta\left({ }^{119} \mathrm{Sn}\right) \mathrm{ppm} ; 113.14$.

$\mathbf{B u}{ }_{3} \mathbf{S n}\left(\mathbf{L}^{1}\right) \quad$ (8): 3 -[N-(4-Nitrophenyl)amido]propenoic acid $(1 \mathrm{~g}, 4.23 \mathrm{mmol})$ was suspended in dry toluene (100 $\mathrm{mL}$ ) and treated with triethylamine $(0.59 \mathrm{~mL}, 4.23 \mathrm{mmol})$. The mixture was refluxed for 2-3 hours. To a solution of triethylammonium 3-[N-(4-nitrophenyl)amido]propenoate in dry toluene, trimethyltin chloride $(4.23 \mathrm{mmol})$ was added as a solid to a reaction flask with constant stirring and reaction mixture was refluxed for $8-10$ hours. The reaction mixture contained $\mathrm{Et}_{3} \mathrm{NHCl}$ was filtered off such that filtrate had the organotin(IV) derivative. The solvent was removed through rotary apparatus under reduced pressure. The mass left behind was recrystallized from $\mathrm{CHCl}_{3}$ and pet.ether (1 : 1). Yield $89 \%$, m.p. $79-80{ }^{\circ} \mathrm{C}$. Analysis: Calculated for $\mathrm{C}_{32} \mathrm{H}_{34} \mathrm{~N}_{2} \mathrm{O}_{5} \mathrm{Sn}: \mathrm{C}, 50.28 ; \mathrm{H}, 6.47 ; \mathrm{N}, 5.33$. Found: $\mathrm{C}, 50.31$; $\mathrm{H}, 6.38$; N, 5.40 .

'H NMR ( $\left.\mathrm{CDCl}_{3}, \mathrm{ppm}\right), 8.06-8.10 \mathrm{~d}(9.08) ; 8.20-8.24 \mathrm{~d}$ (9.08) $(-\mathrm{CH}=\mathrm{CH}-), 7.79-7.85 \mathrm{~m}\left(\mathrm{NO}_{2}-\mathrm{C}_{6} \mathrm{H}_{4}\right), 6.79 \mathrm{~s}(-\mathrm{NH})$, $0.89 \mathrm{t}, 1.27-1.69 \mathrm{~m}\left(\mathrm{Sn}-\mathrm{C}_{4} \mathrm{H}_{9}\right) .{ }^{13} \mathrm{C}$ NMR $\left(\mathrm{CDCl}_{3}, \mathrm{ppm}\right)$, 136.9 (C-1), 130.3 (C-2/6), 134.7 (C-3/5), 126.4 (C-4), 167.2 (C-7), 124.9 (C-8), 113.6 (C-9), 176.8 (C-10), 29.6 (C11), 27.9 (C-12), 16.8 (C-13), 13.6 (C-14). IR ( $\left.\mathrm{KBr}, \mathrm{cm}^{-1}\right)$, $3366 \mathrm{~s} v(\mathrm{NH}), 1710 \mathrm{~s} v(\mathrm{C}=\mathrm{O}), 1587 \mathrm{~s} v\left(\mathrm{COO}_{24 \mathrm{sm}}\right), 1430 \mathrm{~s} v$ $\left(\mathrm{COO}_{\mathrm{syn}}\right)$, Dn $142,575 \mathrm{~m} v(\mathrm{Sn}-\mathrm{C}), 430 \mathrm{w} v(\mathrm{Sn}-\mathrm{O})$. Mass data $m / z(\%)$; R2SnOOCR' 469 (100), RSnOOCR' 411 (n.o), SnOOCR' 351 (2), $\mathrm{R}_{3} \mathrm{Sn}^{+} 291$ (4), $\mathrm{R}_{2} \mathrm{Sn}^{+} 233(7), \mathrm{C}_{6} \mathrm{H}_{5}{ }^{+} 76$ (9), [Sn] ${ }^{+} 120(3), \mathrm{C}_{7} \mathrm{H}_{5} \mathrm{O}_{3} \mathrm{~N}^{+} 165$ (n.o), $\mathrm{CNHO}^{+} 43$ (22), $\left[\mathrm{C}_{6} \mathrm{H}_{5}\right]^{+} 77(9), \mathrm{C}_{6} \mathrm{H}_{5} \mathrm{~N}_{2} \mathrm{O}_{2}{ }^{+} 138(13),\left[\mathrm{C}_{4} \mathrm{H}_{9}\right]^{+} 57(4) . \delta$ ("'Sn) ppm; 59.5 .

$\mathrm{Ph}_{3} \mathrm{Sn}\left(\mathbf{L}^{1}\right)$ (9): 3-[N-(4-Nitrophenyl)amido]propenoic acid $(1 \mathrm{~g}, 4.23 \mathrm{mmol})$ was suspended in dry toluene $(100$ 
$\mathrm{mL})$ and treated with triethylamine $(0.59 \mathrm{~mL}, 4.23 \mathrm{mmol})$. The mixture was refluxed for 2-3 hours. To a solution of triethylammonium 3-[N-(4-nitrophenyl)amido]propenoate in dry toluene, trimethyltin chloride $(4.23 \mathrm{mmol})$ was added as a solid to a reaction flask with constant stirring and reaction mixture was refluxed for $8-10$ hours. The reaction mixture contained $\mathrm{Et}_{3} \mathrm{NHCl}$ was filtered off such that filtrate had the organotin(IV) derivative. The solvent was removed through rotary apparatus under reduced pressure. The mass left behind was recrystallized from $\mathrm{CHCl}_{3}$ and pet.ether $(1$ : 1). Yield $87 \%$, m.p. $63{ }^{\circ} \mathrm{C}$. Analysis: Calculated for $\mathrm{C}_{28} \mathrm{H}_{23} \mathrm{~N}_{2} \mathrm{O}_{5} \mathrm{Snl}: \mathrm{C}, 57.33 ; \mathrm{H}, 3.92 ; \mathrm{N}, 4.77$. Found: C, 57.41; $\mathrm{H}, 3.96 ; \mathrm{N}, 4.71$.

${ }^{1} \mathrm{H}$ NMR $\left(\mathrm{CDCl}_{3}, \mathrm{ppm}\right), 8.02-8.05 \mathrm{~d}(8.98) ; 8.29-8.33 \mathrm{~d}$ (8.98) $(-\mathrm{CH}=\mathrm{CH}-), 7.65-7.66 \mathrm{~m}\left(\mathrm{NO}_{2}-\mathrm{C}_{6} \mathrm{H}_{4}\right), 6.88 \mathrm{~s}(-\mathrm{NH})$, 7.44-7.45m $\left(\mathrm{C}_{6} \mathrm{H}_{5}\right) .{ }^{13} \mathrm{C}$ NMR $\left(\mathrm{CDCl}_{3}, \mathrm{ppm}\right), 137.2(\mathrm{C}-1)$, 130.5 (C-2/6), 134.5 (C-3/5), 126.3 (C-4), 167.2 (C-7), 124.9 (C-8), 114.3 (C-9), 174.4 (C-10), 136.5 (C-11), 135.7 $(\mathrm{C}-12), 129.1(\mathrm{C}-13), 125.4(\mathrm{C}-14) . \mathbb{R}\left(\mathrm{KBr} \mathrm{cm}^{-1}\right), 3382 \mathrm{~s} v$ $(\mathrm{NH}), 1729 \mathrm{~s} v(\mathrm{C}=\mathrm{O}), 1587 \mathrm{~s} v\left(\mathrm{COO}_{\text {assyn }}\right), 1430 \mathrm{~s} v$ $\left(\mathrm{COO}_{\mathrm{sym}}\right), \Delta v 157,425 \mathrm{~m} v(\mathrm{Sn}-\mathrm{O})$. Mass data $\mathrm{m} / \mathrm{z}(\%)$; R2SnOOCR' 505 (2), RSnOOCR' 429 (3), SnOOCR' 351 (n.o), $\mathrm{R}_{3} \mathrm{Sn}^{+} 347$ (26), $\mathrm{R}_{2} \mathrm{Sn}^{+} 271$ (n.o), $\mathrm{C}_{6} \mathrm{H}_{5}^{+} 76$ (3), [Sn] 120 (4), $\mathrm{C}_{7} \mathrm{H}_{5} \mathrm{O}_{3} \mathrm{~N}^{+} 165$ (n.o), $\mathrm{CNHO}^{+} 43$ (9), $\left[\mathrm{C}_{6} \mathrm{H}_{5}\right]^{+} 77$ (4), $\mathrm{C}_{6} \mathrm{H}_{5} \mathrm{~N}_{2} \mathrm{O}_{2}{ }^{+} 138(100) . \delta\left({ }^{119} \mathrm{Sn}\right) \mathrm{ppm} ;-98.2$.

$\mathrm{Me}_{3} \mathrm{Sn}\left(\mathrm{L}^{2}\right)$ (10): 3-[N-(4-nitro-phenylamido]propanoic acid $(1 \mathrm{~g}, 4.20 \mathrm{mmol})$ was suspended in dry toluene (100 $\mathrm{mL}$ ) and treated with triethylamine $(0.58 \mathrm{~mL}, 4.20 \mathrm{mmol})$. The mixture was refluxed for $2-3$ hours. To a solution of triethylammonium 3-[N-(4-nitrophenyl)amido]propanoate in dry toluene, trimethyltin chloride $(4.20 \mathrm{mmol})$ was added as a solid to a reaction flask with constant stirring and reaction mixture was refluxed for $8-10$ hours. The reaction mixture contained $\mathrm{Et}_{3} \mathrm{NHCl}$ was filtered off such that filtrate had the organotin(IV) derivative. The solvent was removed through rotary apparatus under reduced pressure. The mass left behind was recrystallized from $\mathrm{CHCl}_{3}$ and pet.ether $(1$ : 1). Yield $80 \%$, m.p. $75^{\circ} \mathrm{C}$. Analysis: Calculated for $\mathrm{C}_{33} \mathrm{H}_{18} \mathrm{~N}_{2} \mathrm{O}_{5} \mathrm{Sn}: \mathrm{C}, 38.90 ; \mathrm{H}, 4.48 ; \mathrm{N}, 6.98$. Found: C, 39.11; $\mathrm{H}, 4.45 ; \mathrm{N}, 6.88$.

${ }^{1} \mathrm{H} \mathrm{NMR}\left(\mathrm{CDCl}_{3}, \mathrm{ppm}\right), 8.07-8.10 \mathrm{~d}(9.0) ; 8.34-8.38 \mathrm{~d}(9.0)$ $\left(-\mathrm{CH}_{2}-\mathrm{CH}_{2}-\right), 7.59-7.63 \mathrm{~m}\left(\mathrm{NO}_{2}-\mathrm{C}_{6} \mathrm{H}_{4}\right), 7.28 \mathrm{~s}(-\mathrm{NH}), 0.73 \mathrm{~s}$ [58.2] $\left(\mathrm{Sn}_{-} \mathrm{CH}_{3}\right) .{ }^{13} \mathrm{C} \mathrm{NMR}\left(\mathrm{CDCl}_{3}, \mathrm{ppm}\right), 137.8(\mathrm{C}-1)$, 132.5 (C-2/6), 134.6 (C-3/5), 126.3 (C-4), 167.5 (C-7), 124.3 (C-8), 118.6 (C-9), 175.3 (C-10), -1.3 (C-11).

IR $\left(\mathrm{KBr}, \mathrm{cm}^{-1}\right), 3345 \mathrm{~s} v(\mathrm{NH}), 1760 \mathrm{~s} v(\mathrm{C}=0), 1590 \mathrm{~s} v$ $\left(\mathrm{COO}_{a \mathrm{sym}}\right), 1425 \mathrm{~s} v\left(\mathrm{COO}_{\mathrm{syn}}\right), \Delta v 165,562 \mathrm{w} v(\mathrm{Sn}-\mathrm{C})$, $428 \mathrm{~m} v$ (Sn-O). Mass data $\mathrm{m} / \mathrm{z}(\%) ; \mathrm{R}_{2} \mathrm{SnOOCR}^{\prime} 386(2)$, RSnOOCR' 371 (n.o), SnOOCR' 356 (n.o), $\mathrm{R}_{3} \mathrm{Sn}^{+} 164$ (8), $\mathrm{R}_{2} \mathrm{Sn}^{+} 149$ (n.o), $\mathrm{C}_{6} \mathrm{H}_{5}^{+} 76$ (n.o), [Sn] $]^{+} 120$ (4), $\mathrm{C}_{3} \mathrm{H}_{4} \mathrm{O}^{+} 55$ (7), $\left[\mathrm{R}_{2} \mathrm{SnOOCCH}_{2} \mathrm{CH}_{2}\right]^{+} 220(11), \mathrm{C}_{7} \mathrm{H}_{5} \mathrm{O}_{3} \mathrm{~N}^{+} 165(72)$, $\mathrm{CNHO}^{+} 43$ (7), $\mathrm{C}_{3} \mathrm{H}_{5} \mathrm{NO}^{+} 71$ (18), $\left[\mathrm{C}_{6} \mathrm{H}_{5}\right]^{+} 77$ (3), $\mathrm{C}_{6} \mathrm{H}_{5} \mathrm{~N}_{2} \mathrm{O}_{2}{ }^{+} 138(100) . \delta\left({ }^{19} \mathrm{Sn}\right) \mathrm{ppm} ; 170.7$.

$\mathrm{Bu}_{3} \mathrm{Sn}\left(\mathbf{L}^{2}\right)$ (11): 3-[N-(4-nitro-phenylamido]propanoic acid $(1 \mathrm{~g}, 4.20 \mathrm{mmol})$ was suspended in dry toluene (100 $\mathrm{mL})$ and treated with triethylamine $(0.58 \mathrm{~mL}, 4.20 \mathrm{mmol})$. The mixture was refluxed for $2-3$ hours. To a solution of triethylammonium 3-[N-(4-nitrophenyl)amido]propanoate in dry toluene, tributyltin chloride $(4.20 \mathrm{mmol})$ was added as a solid to a reaction flask with constant stirring and reaction mixture was refluxed for 8-10 hours. The reaction mixture contained $\mathrm{Et}_{3} \mathrm{NHCl}$ was filtered off such that filtrate had the organotin(IV) derivative. The solvent was removed through rotary apparatus under reduced pressure. The mass left behind was recrystallized from $\mathrm{CHCl}_{3}$ and pet.ether $(1: 1)$. Yield $60 \%$, m.p. $132-133{ }^{\circ} \mathrm{C}$. Analysis: Calculated for $\mathrm{C}_{22} \mathrm{H}_{3} \mathrm{~N}_{2} \mathrm{O}_{5} \mathrm{Sn}: \mathrm{C}, 50.09 ; \mathrm{H}, 6.83 ; \mathrm{N}, 5.31$. Found: C, 50.21 ; $\mathrm{H}, 6.91 ; \mathrm{N}, 5.28$.

'H NMR ( $\left.\mathrm{CDCl}_{3}, \mathrm{ppm}\right), 8.05-8.09 \mathrm{~d}(9.0) ; 8.33-8.36 \mathrm{~d}(9.0)$ $\left(-\mathrm{CH}_{2}-\mathrm{CH}_{2}-\right), 7.59-7.71 \mathrm{~m}\left(\mathrm{NO}_{2}-\mathrm{C}_{6} \mathrm{H}_{4}\right), 7.28 \mathrm{~s}(-\mathrm{NH}), 0.89 \mathrm{t}$, 1.26-1.67m $\left(\mathrm{Sn}_{-} \mathrm{C}_{4} \mathrm{H}_{9}\right) .{ }^{13} \mathrm{C}$ NMR $\left(\mathrm{CDCl}_{3}, \mathrm{ppm}\right), 137.5(\mathrm{C}-$ 1), 132.9 (C-2/6), 134.7 (C-3/5), 126.5 (C-4), 167.9 (C-7), 124.9 (C-8), 118.7 (C-9), 175.6 (C-10), 29.6 (C-11), 27.7 (C12), 26.8 (C-13), 13.5 (C-14).

IR $\left(\mathrm{KBr}, \mathrm{cm}^{-1}\right), 3356 \mathrm{~s} v(\mathrm{NH}), 1701 \mathrm{~s} v(\mathrm{C}=\mathrm{O}), 1558 \mathrm{~s}$ $v\left(\mathrm{COO}_{3 \mathrm{sim}}\right), 1379 \mathrm{~s} v\left(\mathrm{COO}_{\mathrm{sym}}\right), \Delta v 179,520 \mathrm{~m} v(\mathrm{Sn}-\mathrm{C})$, 433w $v$ (Sn-O). Mass data $\mathrm{m} / \mathrm{z}(\%) ; \mathrm{R}_{2} \mathrm{SnOOCR}^{\prime} 470(17)$, RSnOOCR' 413 (2), SnOOCR' 356 (6), R33 $\mathrm{Sn}^{+} 290$ (14), $\mathrm{R}_{2} \mathrm{Sn}^{+} 233(41), \mathrm{C}_{6} \mathrm{H}_{5}{ }^{+} 76(2),\left[\mathrm{Sn}^{+} 120(13), \mathrm{C}_{3} \mathrm{H}_{4} \mathrm{O}^{+}\right.$ $55(49)$, $\left.\mathrm{R}_{2} \mathrm{SnOOCCH} \mathrm{CH}_{2}\right]^{+} 220(14), \mathrm{C}_{7} \mathrm{H}_{5} \mathrm{O}_{3} \mathrm{~N}^{+} 165(18)$, $\mathrm{CNHO}^{+} 43(47), \mathrm{C}_{3} \mathrm{H}_{5} \mathrm{NO}^{+} 71(19),\left[\mathrm{C}_{6} \mathrm{H}_{5}\right]^{+} 77$ (11), $\mathrm{C}_{6} \mathrm{H}_{5} \mathrm{~N}_{2} \mathrm{O}_{2}{ }^{+} 138(17), \mathrm{C}_{4} \mathrm{H}_{9}{ }^{+} 57(100) . \delta\left({ }^{19} \mathrm{Sn}\right) \mathrm{ppm}$; 157.2.

$\mathrm{Ph}_{3} \mathrm{Sn}\left(\mathbf{L}^{2}\right)$ (12): 3-[N-(4-nitro-phenylamido]propanoic acid $(1 \mathrm{~g}, 4.20 \mathrm{mmol})$ was suspended in dry toluene $(100$ $\mathrm{mL}$ ) and treated with triethylamine $(0.58 \mathrm{~mL}, 4.20 \mathrm{mmol})$. The mixture was refluxed for $2-3$ hours. To a solution of triethylammonium 3-[N-(4-nitrophenyl)amido]propanoate in dry toluene, triphenyltin chloride $(4.20 \mathrm{mmol}$ ) was added as a solid to a reaction flask with constant stirring and reaction mixture was refluxed for $8-10$ hours. The reaction mixture contained $\mathrm{Et}_{3} \mathrm{NHCl}$ was filtered off such that filtrate had the organotin(IV) derivative. The solvent was removed through rotary apparatus under reduced pressure. The mass left behind was recrystallized from $\mathrm{CHCl}_{3}$ and pet.ether (1 : 1). Yield $88 \%$, m.p. $105{ }^{\circ} \mathrm{C}$. Analysis: Calculated for $\mathrm{C}_{28} \mathrm{H}_{24} \mathrm{~N}_{2} \mathrm{O}_{5} \mathrm{Snn}: \mathrm{C}, 57.24 ; \mathrm{H}, 4.08 ; \mathrm{N}, 4.77$. Found: C, 57.29; $\mathrm{H}, 4.02 ; \mathrm{N}, 4.73$.

'H NMR ( $\left.\mathrm{CDCl}_{3}, \mathrm{ppm}\right),(8.07-8.10 \mathrm{~d}(9.0) ; 8.32-8.34 \mathrm{~d}$ (9.0) $\left(-\mathrm{CH}_{2}-\mathrm{CH}_{2}-\right), 7.61-7.65 \mathrm{~m}\left(\mathrm{NO}_{2}-\mathrm{C}_{6} \mathrm{H}_{4}\right), 7.27 \mathrm{~s}(-\mathrm{NH})$, 7.43-7.44m $\left(\mathrm{Sn}_{-} \mathrm{C}_{6} \mathrm{H}_{5}\right) .{ }^{13} \mathrm{C}$ NMR $\left(\mathrm{CDCl}_{3}, \mathrm{ppm}\right), 137.3(\mathrm{C}-$ 1), 132.2 (C-2/6), 134.8 (C-3/5), 126.3 (C-4), 167.4 (C-7), 124.6 (C-8), 118.9 (C-9), 177.6 (C-10), 136.1 (C-11), 135.8 (C-12), 129.1 (C-13), 125.4 (C-14).

IR $\left(\mathrm{KBr}, \mathrm{cm}^{-1}\right), 3386 \mathrm{~s} v(\mathrm{NH}), 1720 \mathrm{~s} v(\mathrm{C}=\mathrm{O}), 1599 \mathrm{~s} v$ $\left(\mathrm{COO}_{\text {assm }}\right), 1429 \mathrm{~s} v\left(\mathrm{COO}_{\mathrm{ssm}}\right), \Delta v 170,446 \mathrm{~m} v$ (Sn-O). Mass data $\mathrm{m} / \mathrm{z}(\%)$; R2 SnOOCR' 510 (n.o), RSnOOCR' 433 (n.o), SnOOCR' 356 (20), $\mathrm{R}_{3} \mathrm{Sn}^{+} 347$ (53), $\mathrm{R}_{2} \mathrm{Sn}^{+} 271$ (12),

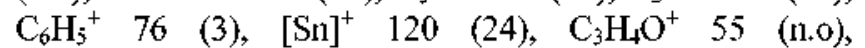
$\left[\mathrm{R}_{2} \mathrm{SnOOCCH}_{2} \mathrm{CH}_{2}\right]^{+} 220$ (n.o), $\mathrm{C}_{7} \mathrm{H}_{3} \mathrm{O}_{3} \mathrm{~N}^{+} 165$ (n.o), $\mathrm{CNHO}^{+} 43(3), \mathrm{C}_{3} \mathrm{H}_{5} \mathrm{NO}^{+} 71(5),\left[\mathrm{C}_{6} \mathrm{H}_{5}\right]^{+} 77(100)$, $\mathrm{C}_{6} \mathrm{H}_{5} \mathrm{~N}_{2} \mathrm{O}_{2}{ }^{+} 138(21) . \delta\left({ }^{119} \mathrm{Sn}\right)$ ppm; -46.7 .

General chemical reactions are given in Scheme 1 and numbering for NMR is given in Scheme 2 . 


$$
\begin{aligned}
& \mathrm{HL}^{1}: \quad \mathrm{NO}_{2} \bigcirc-\stackrel{\mathrm{O}}{\longrightarrow}-\stackrel{\mathrm{NH}}{\mathrm{C}}-\mathrm{CH}=\mathrm{CH}-\stackrel{\mathrm{C}}{-}-\mathrm{OH} \\
& \mathrm{HL}^{2}: \quad \mathrm{NO}_{2} \bigcirc-\stackrel{\mathrm{O}}{\mathrm{NH}}-\stackrel{\mathrm{C}}{\mathrm{C}}-\mathrm{CH}_{2}-\mathrm{CH}_{2}-\stackrel{\mathrm{O}}{\mathrm{C}}-\mathrm{OH} \\
& \left(\mathrm{NO}_{2}-\bigcirc-\mathrm{NH}-\stackrel{\mathrm{O}}{\mathrm{C}}-\mathrm{R}-\mathrm{I}-\mathrm{C}-\mathrm{O}\right)_{2}-\mathrm{SnR}_{2}^{\prime}
\end{aligned}
$$

\begin{tabular}{|c|c|c|}
\hline Comp. No. & $\mathrm{R}$ & $\mathrm{R}^{\prime}$ \\
\hline$(\mathbf{1})$ & $-\mathrm{CH}=\mathrm{CH}-$ & $\mathrm{Me}\left(\mathrm{CH}_{3}\right)$ \\
\hline$(\mathbf{2})$ & $-\mathrm{CH}=\mathrm{CH}-$ & $\mathrm{Ph}\left(\mathrm{C}_{6} \mathrm{H}_{5}\right)$ \\
\hline$(\mathbf{3})$ & $-\mathrm{CH}=\mathrm{CH}-$ & $\operatorname{Oct}\left(\mathrm{C}_{8} \mathrm{H}_{17}\right)$ \\
\hline$(\mathbf{4})$ & $-\mathrm{CH}_{2}-\mathrm{CH}_{2}-$ & $\operatorname{Me}\left(\mathrm{CH}_{3}\right)$ \\
\hline$(\mathbf{5})$ & $-\mathrm{CH}_{2}-\mathrm{CH}_{2}-$ & $\operatorname{Bu}\left(\mathrm{C}_{4} \mathrm{H}_{9}\right)$ \\
\hline$(\mathbf{6})$ & $-\mathrm{CH}_{2}-\mathrm{CH}_{2}-$ & $\operatorname{Oct}\left(\mathrm{C}_{8} \mathrm{H}_{17}\right)$ \\
\hline
\end{tabular}

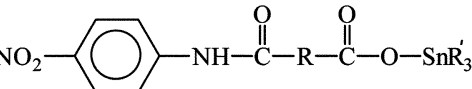

\begin{tabular}{|c|c|c|}
\hline Comp. No. & $\mathrm{R}$ & $\mathrm{R}^{\prime}$ \\
\hline $\mathbf{( 7 )}$ & $-\mathrm{CH}=\mathrm{CH}-$ & $\mathrm{Me}\left(\mathrm{CH}_{3}\right)$ \\
\hline$(\mathbf{8})$ & $-\mathrm{CH}=\mathrm{CH}-$ & $\mathrm{Bu}\left(\mathrm{C}_{4} \mathrm{H}_{9}\right)$ \\
\hline$(\mathbf{9})$ & $-\mathrm{CH}=\mathrm{CH}-$ & $\mathrm{Ph}\left(\mathrm{C}_{6} \mathrm{H}_{5}\right)$ \\
\hline$(\mathbf{1 0})$ & $-\mathrm{CH}_{2}-\mathrm{CH}_{2}-$ & $\mathrm{Me}\left(\mathrm{CH}_{3}\right)$ \\
\hline$(\mathbf{1 1})$ & $-\mathrm{CH}_{2}-\mathrm{CH}_{2}-$ & $\mathrm{Bu}\left(\mathrm{C}_{4} \mathrm{H}_{9}\right)$ \\
\hline$(\mathbf{1 2})$ & $-\mathrm{CH}_{2}-\mathrm{CH}_{2}-$ & $\mathrm{Ph}\left(\mathrm{C}_{6} \mathrm{H}_{5}\right)$ \\
\hline
\end{tabular}

Scheme 1

$$
\mathrm{R}_{2} \mathrm{SnO}+2 \mathrm{HL} \underset{\text { Retlux } 8-10 \mathrm{hrs} .}{\stackrel{\text { Toluene }}{\longrightarrow}} \mathrm{R}_{2} \mathrm{SnL}_{2}+\mathrm{H}_{2} \mathrm{O}
$$

$\mathrm{R}_{2} \mathrm{SnCl}_{2}+2 \mathrm{E} 1_{3} \mathrm{NI} 1 \mathrm{~L} \frac{\text { Toluene }}{\text { Reflux } 8-10 \text { hrs. }}$

$$
\mathrm{R}_{2} \mathrm{SnL}_{2}+2 \mathrm{Et}_{3} \mathrm{NHCl}
$$

$\mathrm{R}_{3} \mathrm{SnCl}+\mathrm{Et}_{3} \mathrm{NHL} \underset{\text { Reflux 8-10 hrs. }}{\stackrel{\text { Toluene }}{\longrightarrow}} \mathrm{R}_{3} \mathrm{SnL}+\mathrm{Et}_{3} \mathrm{NHCl}$

$\mathrm{HL}=\mathrm{HL}^{1}$ and $\mathrm{HL}^{2}$

\section{Results and Discussion}

With few exceptions all the complexes are coloured solids.<smiles>[M]OC([R])=O</smiles><smiles></smiles>

2<smiles></smiles>

3

Scheme 3. Possible coordination modes of carboxylate group to metal.

They are slightly soluble in cold methanol and $n$-hexane but freely soluble in $\mathrm{DMSO}$ and $\mathrm{CHCl}_{3}$. The complexes have sharp melting points. The metal derivatives are stable at room temperature and are non-hygroscopic.

Infrared Spectra. The FTIR data are consistent with the formation of compounds with the composition $\mathrm{R}_{2} \mathrm{SnL}_{2}$ and $\mathrm{R}_{3} \mathrm{SnL}$. The carboxylate group ligands coordinate to metal ions by different modes as shown in Scheme 3.34

Disappearance of a broad band in the spectra of the complexes in the region $2800-2200 \mathrm{~cm}$ ', which was present in free ligands as a weak intensity band, suggests the deprotonation of the free $\mathrm{COOH}$ group upon complexation.

The presenee of $\mathrm{Sn}-\mathrm{C}$ absorption bands in all complexes in the region $520-580 \mathrm{~cm}^{1}$ confirms the formation of $\mathrm{Sn}-\mathrm{C}$ bond in all complexes.

The appearance of very weak and wide band in the region of $405-480 \mathrm{~cm}$ ' assigned to $v_{\text {ass }}(\mathrm{Sn}-\mathrm{O})$, indicate the formation of $\mathrm{Sn}-\mathrm{O}$ bond.

The carboxylate group generally adopts a bridged structure in the solid state unless the organic substituents at the tin atom are bulky or the carboxylate group is branched at $\alpha$ carbon. ${ }^{35}$ The IR absorption spectra indicate that $v_{\text {asy }}(\mathrm{COO})$ values $\left(1520-1599 \mathrm{~cm}^{1}\right)$ and $v_{\mathrm{sim}}(\mathrm{COO})$ values (1379-1465 $\mathrm{cm}$ ') reveal the strong interaction between the oxygens and the tin atom. The magnitude of the $\left(v_{\text {assm }}-v_{\text {sim }}\right) C O O=(\Delta v)$ separation, which has been shown to be useful in identifying structural features, lies in the range 142-182 $\mathrm{cm}^{1}$ which confirm the bidentate nature of the ligand. ${ }^{36}$

Mass Spectrometry. Molecular ion peak was exceptionally observed in some of the complexes. All compounds lose an alky or aryl group first, followed by elimination of $\mathrm{CO}_{2}$ and other neutral species which ultimately gives $\mathrm{C}_{6} \mathrm{H}_{5}^{-}$. Another possible route is disintegration of the ligand and stepwise elimination of $\mathrm{R}$ groups to give $\mathrm{Sn}^{-}$as the end product.

Base peak in the compounds (1)-(3) is due to fragment $\left[\mathrm{C}_{6} \mathrm{H}_{5} \mathrm{O}_{2} \mathrm{~N}_{2}\right]^{+}$at $\mathrm{m} / \mathrm{z} 138$ (100), while in compounds (4), (5)<smiles>[R][Sn]([R])([R])OC(=O)C=CC(=O)Nc1ccc([N+](=O)[O-])cc1</smiles><smiles>[R][Si]([R])([R])OC(=O)CCCC(=O)Nc1ccc([N+](=O)[O-])cc1</smiles>

$$
\begin{aligned}
& -\stackrel{\mathrm{C}}{\mathrm{C}} \mathrm{H}_{3} \\
& \stackrel{11}{\mathrm{C}} \stackrel{12}{\mathrm{CH}_{2}}-\mathrm{CH}_{2}-\stackrel{1.3}{\mathrm{C}} \mathrm{CH}_{2}-\stackrel{1+}{\mathrm{C}} \mathrm{H}_{3} \\
& -\stackrel{11}{\mathrm{C}} \mathrm{H}_{2}-\stackrel{12}{\mathrm{C}} \mathrm{H}_{2}-\stackrel{13}{\mathrm{C}} \mathrm{H}_{2}-\stackrel{14}{\mathrm{C}} \mathrm{H}_{2}-\stackrel{15}{\mathrm{C}} \mathrm{H}_{2}-\stackrel{16}{\mathrm{C}} \mathrm{H}_{2}-\stackrel{1 ?}{\mathrm{C}} \mathrm{H}_{2} \stackrel{18}{\mathrm{C}} \mathrm{H}_{3} \\
& \bigcirc_{14}^{13}
\end{aligned}
$$


and (6), it is due to $\left[\mathrm{R}_{2} \mathrm{SnOOCCH}_{2} \mathrm{CH}_{2}\right]^{+}$at $\mathrm{m} / \mathrm{z} .220(100)$, $\left[\mathrm{C}_{4} \mathrm{H}_{9}\right]^{+}$at $\mathrm{m} / \mathrm{z} .57(100),\left[\mathrm{CH}_{2} \mathrm{CH}_{2} \mathrm{CO}^{-}\right.$at $\mathrm{m} / \mathrm{z} .55(100)$, respectively. In triorganotin(IV) derivatives, (7)-(12), base peak is duc to fragment [SnOOCR'] at $\mathrm{m} / \mathrm{z} .356(100)$, [ $\mathrm{R}_{2} \mathrm{SnOOCR}$ '] at $\mathrm{m} / \mathrm{7} .469(100)$, [SnOOCR'] at $\mathrm{m} / \mathrm{z} .351$ (100), $\left[\mathrm{NHC}_{6} \mathrm{H}_{4} \mathrm{NO}_{2}\right]^{+}$at $\mathrm{m} / 7.138(100),\left[\mathrm{C}_{4} \mathrm{H}_{4}\right]^{+}$at $\mathrm{m} / 7.57$ (100) and $\left[\mathrm{C}_{6} \mathrm{H}_{5}\right]^{+}$at $\mathrm{m} / 7.77(100)$, respectively. General fragmentation patterns are given as Schemes 4 and 5 .

Nuclear Magnetic Resonance Spectra. The 'H NMR spectra of the complexes exhibit the useful features and observed chemical shifts are reported in Txperimental Section.

In studied complexes, the $\mathrm{CO}(\mathrm{OH})$ resonanee of the ligands is absent which suggests the replacement of the carboxylic proton by the organotin(IV) moicty. ${ }^{3} J\left({ }^{1} \mathrm{H},{ }^{1} \mathrm{H}\right)$ values for HL' compounds (1)-(3) and (7)-(9) suggests that protons of acetylene group $(\mathrm{HC}=\mathrm{HC})$ are in the cis position. In all diorganotin(IV) and triorganotin(IV) derivatives, the -NH resonanee observed as a broad or a sharp weak signal. The aromatic carbon resonanes were assigned by comparing experimental chemical shifts with those calculated by the incremental method. ${ }^{37}$ In triorganotin carboxylates $\left.\left.{ }^{2} J\right]^{119} \mathrm{Sn}-{ }^{-} \mathrm{H}\right]$ for compounds (7) and (8) suggests tetrahedral geonetry (Fig. 1(a)) around the tin atom. Unlike the triorganotin carboxylates in solution, the geometry of diorganotin dicarboxylates eannot be defined with certainty because of dynamic process involving in carboxylate oxygens due to competition in their coordination behaviour with the tin atom, ${ }^{38}$ However, in the solid state the tin atom is mostly

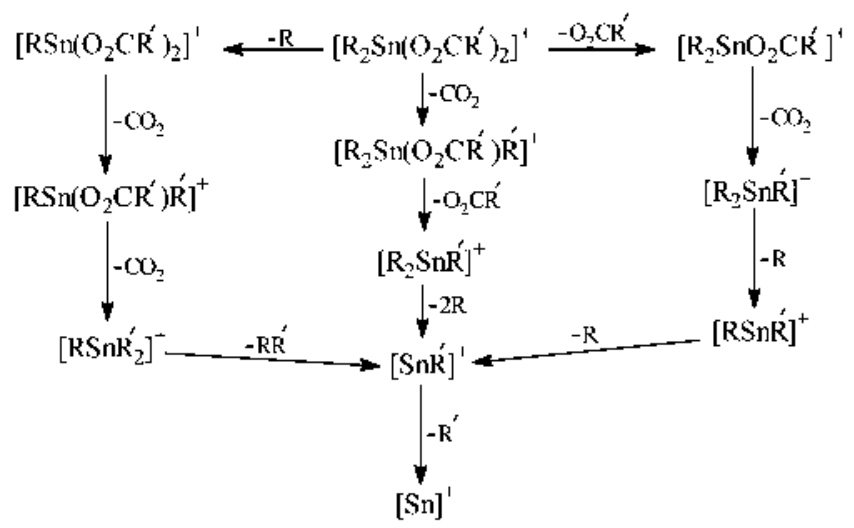

Scheme 4. General fragmentation pattern for $\mathrm{R}_{2} \mathrm{SnL}_{2}{ }^{1} / \mathrm{R}_{2} \mathrm{SnL}_{2}^{2}$.

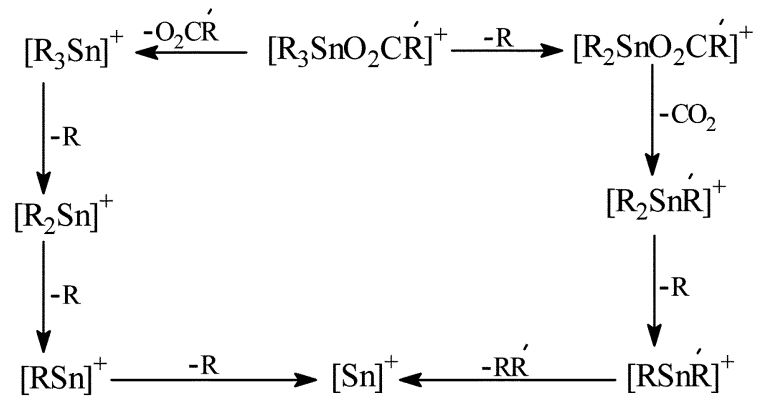

Scheme 5. General fragmentation pattern for $\mathrm{R}_{3} \mathrm{SnL}^{1} / \mathrm{R}_{3} \mathrm{~S}_{n} \mathrm{~L}^{2}$.<smiles>[R][Sn]([R])([R])OC(C)=O</smiles>

(a)

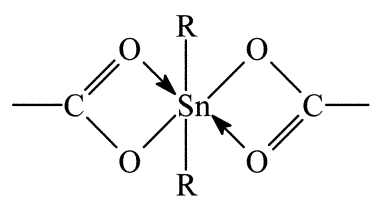

(b)
Figure 1. Suggested structures of the complexes.

hexa-coordinated in such systems (Fig. l(b)). ${ }^{39}$

The ${ }^{13} \mathrm{C}$. NMR characteristic resonance peaks of the complexes along with the ligands, recorded in $\mathrm{CDCl}_{3}$ are presented in Tixperimental Section.

The speetra of the organotin(IV) derivatives are consistent with the following observations:

- The resonanee of the carboxylic earbon (C-10) in all the compounds move to lower field as compared with the ligands, suggesting the coordination of the ligand, through carboxylic oxygen, to the organotin(IV) moicty.

- The carbon of phenyl and alkyl groups attached to tin are observed at almost similar positions in the experimental data as calculated from incremental method ${ }^{38}$ and reported in the literature. ${ }^{28-32}$

In all complexes, ${ }^{119} \mathrm{~S}$ spectrat show only a sharp singlet indicating the formation of single species. Tn general ${ }^{119} \mathrm{~S}$ chemical shifts move to lower frequency with inereasing coordination number. Although the shift ranges are somewhat dependent on the nature of the substituents at the tir

Table 1. Brine Shrimp (Artemia Salina) Lethality Bioassay ${ }^{3}$ for $\mathrm{R}_{2} \mathrm{SnL}_{2} / \mathrm{R}_{2} \mathrm{SnL}_{2}{ }^{2}$ and $\mathrm{R}_{3} \mathrm{SnL}^{1} / \mathrm{R}_{3} \mathrm{SnL}^{2}$

\begin{tabular}{ccccc}
\hline $\begin{array}{c}\text { Comp. } \\
\text { No. }\end{array}$ & $\begin{array}{c}\text { Dose } \\
(\mu g / 17 \mathrm{~L})\end{array}$ & $\begin{array}{c}\text { No. of } \\
\text { Shrimps }\end{array}$ & $\begin{array}{c}\text { No. of } \\
\text { Survivors }\end{array}$ & $\begin{array}{c}\mathrm{LD}_{S \mathrm{ct}} \\
(\mu \mathrm{g} / \mathrm{mL})\end{array}$ \\
\hline III. & 100 & 30 & 5 & 74.16 \\
& 10 & 30 & 9 & \\
(1) & 1 & 30 & 10 & \\
& 100 & 30 & 0 & $<1$ \\
& 10 & 30 & 0 & \\
(2) & 1 & 30 & 0 & \\
& 100 & 30 & 0 & 7.14 \\
& 10 & 30 & 10 & \\
(3) & 1 & 30 & 30 & \\
& 100 & 30 & 10 & - \\
& 1 & 30 & 10 & \\
(7) & 100 & 30 & 10 & \\
& 10 & 30 & 10 & - \\
& 1 & 30 & 10 & \\
(8) & 100 & 30 & 10 & \\
& 10 & 30 & 0 & 25.94 \\
& 1 & 30 & 10 & \\
(9) & 100 & 30 & 0 & 1.90 \\
& 10 & 30 & 1 & \\
& 1 & 30 & 9 & \\
\hline
\end{tabular}

Standard drug: Etoposide

I.I) ${ }_{50}(\mu \mathrm{g} / \mathrm{mL}) ;$. 


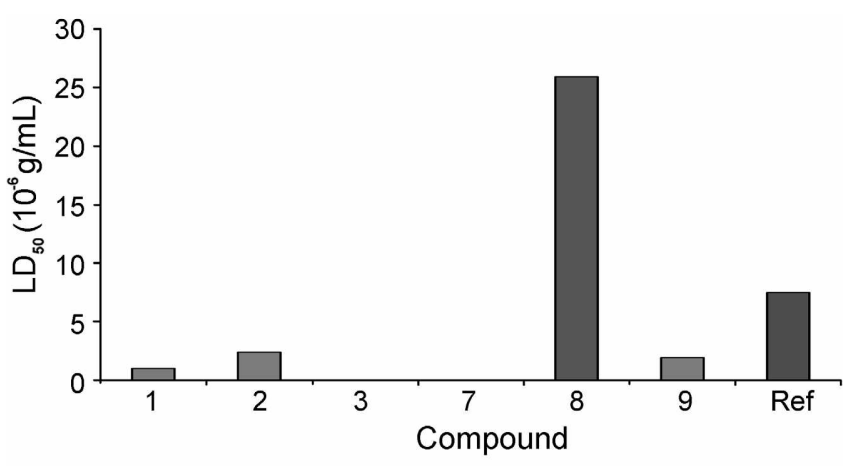

Figure 2. Cytotoxic study ol organotin(IV) derivatives.

atom. In all the complexes, ${ }^{1 / 4} \mathrm{Sn}$ chemical shifts values lie in the tetrahedral environment around the lin atom in noncoordinated solvents.

Biological Activity: $\mathrm{LD}_{50}$ data of the compound (1)-(3) and (7)-(9) have been detemined by the Brine-Shrimp assay method $^{40}$ and the results are summarized in Table $I$ and Figure 2. Previous reports ${ }^{31 .+2}$ show that the nature of organic group is responsible for the toxicity of organotin compounds. The highest toxicity being shown by the ligand with $\mathrm{LD}_{50}$ value $74.16 \mu \mathrm{g} / \mathrm{mL}$ while the compounds (3) and (7) do not show any toxicity al all. Antibacterial activity tests of compounds (1)-(3) and (7)-(9) were carried out against various bacteria by the "agar diffusion technique". The screening tests show that phenyltin carboxylates are the most potent candidates against the tested bacteria. The activity of the other derivatives varies according to their $R$ groups. I Iowever, all of these compounds are aclive against Bacillas subrilis. The results are given in Table 2 and Figure 3.

Compounds (1)-(11) were also tested for their antilungal

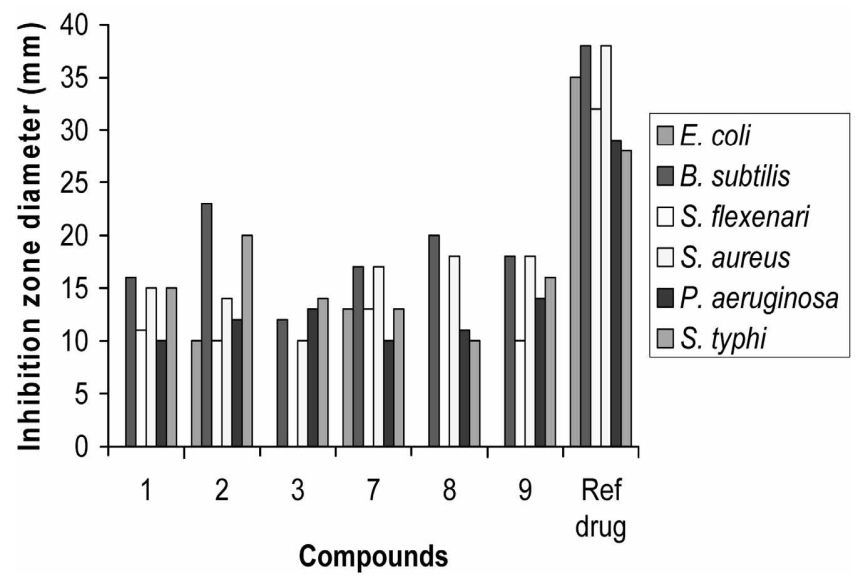

Figure 3. Antibacterial aclivity of organolin(IV) derivatives against various hacieria.

activity by the tube diflusion method. ${ }^{+4}$ The results of antifungal assay are given in Table 3 and Figure 4 . It has been shown that the ligand along with its organotin carboxylates are significantly active against all of the tested fungal strains. It has been reported that, within the given series, the triorganotin(IV) derivatives are more active against fingi ${ }^{45}$ as compared to diorganotin(IV) derivalives. The insecticidal activity data of the compounds (4)-(6) and (10)-(12) are given in Table 4 and Figure 5. It shows that diorganotin(IV) dicarboxylates are found to be inactive against the tested insects while the triorganotin(IV) carboxylates shows the activity. It can be explained as the length or number of $\mathrm{R}$ group increases, activity also increases. That's why triorganotin(IV) derivatives show more activity as compared to diorganotin derivatives and butyl derivatives show more activity than methyl derivatives.

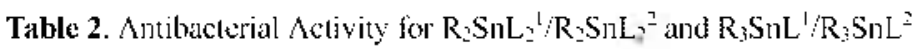

\begin{tabular}{|c|c|c|c|c|c|c|c|c|}
\hline \multirow{2}{*}{ Vame of Bacteria } & \multicolumn{7}{|c|}{ Compound No. } & \multirow{2}{*}{ Slandard Drug } \\
\hline & III. & (1) & (2) & (3) & (7) & (8) & (9) & \\
\hline Escherichia coli & 11 & - & 10 & - & 13 & - & - & Ampiciline $\left(\mathrm{I}_{2} \mathrm{O}\right)_{3} \mathrm{Ccphalexin \textrm {Na }}$ \\
\hline Bacillus subtilis & 17 & 16 & 23 & 12 & 17 & 20 & 18 & $=$ \\
\hline Shigella flerenari & 11 & 11 & 10 & - & 13 & - & 10 & $\cdot$ \\
\hline Stephlococcus aterews & 14 & 1.5 & 14 & 10 & 17 & 18 & 18 & * \\
\hline Pseudomonoas aeruginosa & 10 & 10 & 12 & 13 & 10 & 11 & 14 & * \\
\hline Salnondla tiphi & 11 & 1.5 & 20 & 14 & 13 & 10 & 16 & $\cdot$ \\
\hline
\end{tabular}

Table 3. Antilungal Activity tor $\mathrm{R}_{2} \mathrm{SnL}_{2} \mathrm{~L}_{2} / \mathrm{SnL}_{2}{ }^{2}$ and $\mathrm{R}_{3} \mathrm{~S}_{1} \mathrm{~L}^{1} / \mathrm{R}_{3} \mathrm{~S}_{11} \mathrm{~L}^{7}$

\begin{tabular}{|c|c|c|c|c|c|c|c|c|c|c|c|c|c|c|}
\hline \multirow{2}{*}{ Name of Fungi } & \multicolumn{13}{|c|}{ Compound No. } & \multirow{2}{*}{ Standard Drug } \\
\hline & HII.' & $\mathrm{HIL}$ & (1) & (2) & (3) & (4) & (5) & (6) & $(7)$ & $(8)$ & (9) & $(10)$ & (11) & \\
\hline Trichoplinton longifustus & 57.8 & 70 & 78.9 & 45 & 47.3 & 75 & 75 & 75 & 78.9 & 100 & 84.2 & 70 & 70 & Miconazolc, Ketoconazole \\
\hline Candicla albicans & 6.3 .1 & 10 & 0 & 0 & 0 & 10 & 0 & 10 & 68.4 & 100 & 100 & 25 & 0 & Miconazole, Ketoconazole \\
\hline Aspergilhas flavies & 0 & 60 & 0 & 0 & 10 & 60 & 60 & 70 & 0 & 100 & 100 & 70 & 60 & Ampotericin B. Flucylosine \\
\hline Microsporth canis & 40 & 30 & 0 & 40 & 47.3 & 30 & 35 & 40 & 73 & 90 & 88 & 30 & 30 & Miconazolc, Ketoconazole \\
\hline Fissoritum solani & 15 & 30 & 50 & 70 & 0 & 30 & 0 & 10 & 70 & 90 & 80 & 0 & 0 & Ketoconazole \\
\hline Catdida glaberata & 0 & 10 & 0 & 0 & 0 & 10 & 10 & 10 & 0 & 100 & 94.7 & 0 & 0 & Keloconazole \\
\hline
\end{tabular}




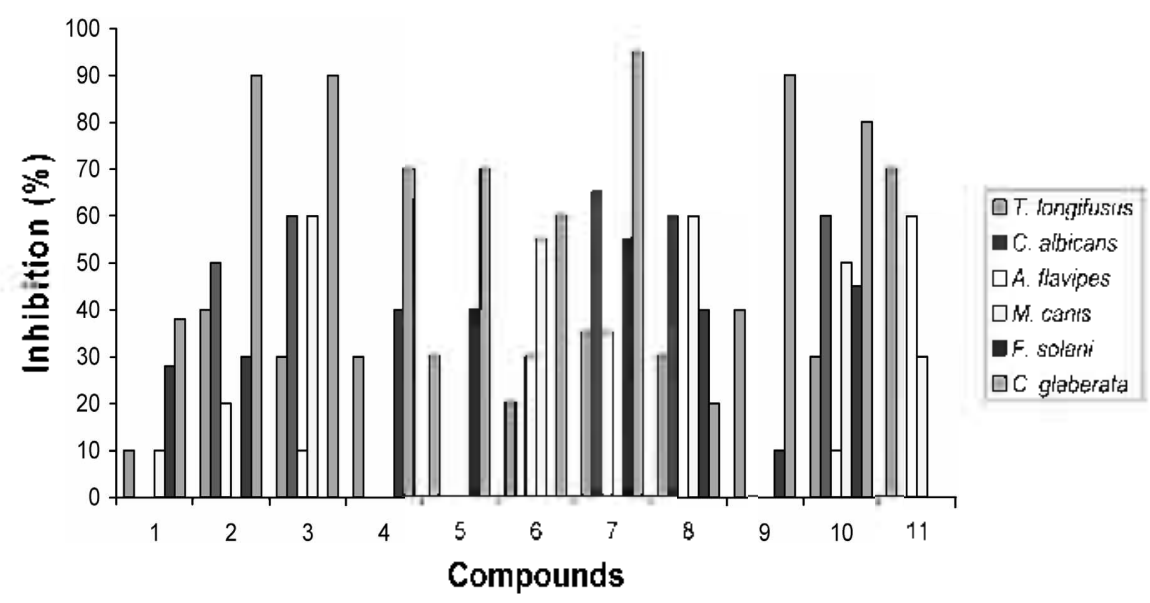

Figure 4. Antifungal activity of organolin( IV) derivatives against various lingi.

Table 4. Insecicidal activity data for $\mathrm{R}_{2} \mathrm{SnL}_{2}$, and $\mathrm{R}_{3} \mathrm{SnL}^{\text {? }}$

\begin{tabular}{|c|c|c|c|c|c|c|c|c|c|}
\hline \multirow{2}{*}{ Vame of Insect } & \multicolumn{7}{|c|}{$\%$ Mortality (-ve Control) } & \multirow{2}{*}{ - ve Control } & \multirow{2}{*}{ Standard Drug } \\
\hline & HII..$^{2}$ & (4) & (5) & (6) & (10) & (11) & $(12)$ & & \\
\hline Tribolitum castanetm & 0 & 0 & 0 & 0 & 20 & 25 & 0 & 100 & Premethrin \\
\hline Sitophinus orizae & 25 & 0 & 0 & 0 & 0 & 25 & 25 & 100 & Premethrin \\
\hline Rhyeopertha dontinica & 0 & 0 & 0 & 0 & 25 & 0 & 0 & 100 & Premethrin \\
\hline Collosbrints analis & 0 & 0 & 0 & 0 & 20 & 25 & 0 & 100 & Premethrin \\
\hline
\end{tabular}

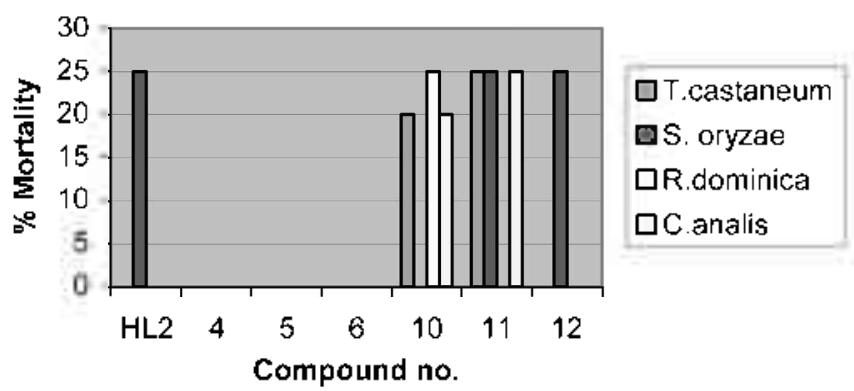

Figure 5 . Insecticidal activity of organolin(IV) derivalives.

\section{Conclusion}

It was concluded that in solid state organotin(IV) carboxylates have live or six coordination around tin, due to bidentate nature of carboxylates group, while in solution the bidentate carboxylate group is cleaved and the resulting monomer contains four coordinated tin with a tetrahedral arrangement. All complexes show the primary lragmentation due to the loss of the alkyl group followed by climination of $\mathrm{CO}_{2}$ and the remaining part of the ligand, which leaves $\mathrm{Sn}$ as the end product. Organotin(IV) carboxylates are found to be active against lested Brine Shrimp larvac, bacteria, lungi and the insects.

Acknowledgment. Financial support from the University Research Fund (URF) of Quaid-i-Azam University is highly acknowledged.

\section{References}

I. ILonnik, W. D.: Zuckemtran, J. J. J. Orgumonef. Chem. 1979, /33, 178.

2. Itarrison, P. G; Philips, R, C. J. Organomet Chem. 1979, 37, 182.

3. Molloy, K. C.; Purcell, T. Gi, Schumann, Il.: Zuckeman, I. J. Organonetallic:s $1986,5,85$.

4. Ilolucek, 3.; Hlandlit, K.; Nadwomik, M.; Lycka, A. J. Organomet Chen 1983, 147, 258 .

5. Ng. S. W.; Wai, C.: Kumar Das, V. G J. Orgonomet. Chen. 1988. 59,345 .

6. Sandhu, G. K.: Verma, S. P.; Ticknik, I:. R. T. J. Organomet. (hem 1990, 195, 393.

7. Tieksik, E. R. T. Appl Oryanomat Chem 1991, 5,1.

8. Ng. S. W.: Kumar Das, V. Gi; Ticknik. E. R. T. $J$. Orgumonet. Chem 1991, $/ /, 403$.

9. Vasta,, , Jain, V, K; [ as, T, K; Ticknik, F., R. T. J. Orgonomet Chem. 1991, 2l, 421 .

10. Teoh, S. Ci; Ang, S. HI.: Looi, E. S.; Keok, C. A.; Teo, S. B.; Furl, II. K. J. Orguthomet Chem 1997, 527, 15 .

11. Sexana, A. K.: I tuber, F. Coord. Chem. Rev. 1989, 95, 109.

12. Barbicri, R. Ihorg. Chim. Acta 1992, 191, 253.

13. Cupta, S. P. Chem. Rev: 1994, 94, 1507.

14. Citelen, M. Coord Chem. Rev 1996, 151,41

15. Vos, D. de; Willem, R.; Giekn, M.; Van Wingerden, K. C.; Nooter, K. Mat. Based Ditugs 19y8, 5.179.

16. Ilolloway, C. F., Melnik, M, Main Grotip Wet Chem 2000, 23, 555 .

17. Tir-Based Anitwhor Drugs; NATO ASl Series, H37: (jiclen, M., lid.: Springer-Vurlas: Burlin, 1990.

I8. Okawa, R.; Webster, D. 1.; Rockow, I. R. J. Am. Chem. Soc $1960,82,3287$.

19. Tiano, L.; Fedcli, D.: Moretti, M.; Falcioni, G. Appl. Orgumomet Chem. 2001, 15, 575 .

20. Gabbianclli, R.; Villarini, Mi; Falcioni, (i.; lupidi, G. Appl. 
Organtontet. Chem. 2002, 16, 163.

21. Gielen, M.; El-Khloufi, A.; Biesemans, M.; Willem, R.; MeunierPiert, J. Pohthedron 1992, $I, 1861$.

22. Gielen, M.; Lelieveled, P; Vos, D. de; Pan, H.; Willem, R.; Biesemans, M.; Fiebig, H. H. Inorg. Chim. Acta 1992, 115, 196.

23. Song, X.; Yang, Z; Xie, Q.; Li, J. J. Organonet, Chem. 1998, 103, 566.

24. Gielen, M.; Biesemans, M.; Vos, D. de; Willem, R. J. Inorg. Biochem. 2000, 79, 139.

25. Camacho-Camacho, C.; Vos, D. de; Mahieu, B.; Gielen, M.; Kemmer, M.; Biesemans, M.; Willem, R, Main Grotp Met. Chem. $2000,23,433$.

26. Nath, M.; Yadav, R.; Gielen, M.; Dalil, H.; Vos, D. de; Eng. G. Appl. Organomet. Chen. 1997, $11,727$.

27. Kemmer, M.; Biesemans, M.; Willem, R. Main Grotp Met. Chem. $2000,23,433$.

28. Masood, M. T.; Ali, S.; Danish, M.; Mazhar, M. Syyth. React. Inorg. Met. $=$ Org. Chem. 2002, 32(1), 9.

29. Ahmed, S.; Ali, S.; Ahmed, F; Bhatti, M. H.; Badshah, A.; Mazhar, M.; Khan, K. M. Synth. React. Inorg, Met.-Org. Chent. 2002, 32(8), 1725 .

30. Marchetti, F.; Pellei, M.; Pettinari, C.; Pettinari, R.; Rivarola, E.; Santini, C.; Skeltom, B. W.; White, A. H. J. Appl. Organomet. Chem. 2005, 690, 1878 .

31. Ali, S.; Khokhar, M. N.; Bhatti, M. H.; Mazhar, M.; Masood, M. T.; Shahid, K.; Badshah, A. Synth. React. Inory, Met.-Org. Chent.
$2002,32(8), 1579$.

32. Ahmad, F.; Ali, S.; Parvez, M.; Munir, A.; Mazhar, M.; Khan, K. M.; Shah, T. A. Heteroatom Chem. 2002, 13(7), 638.

33. Armergo, W. L. F.; Chai, C. L. L. Purfication of Laboratory Chemicals, $5^{\text {th }}$ ed.; Elsevier. USA, 2003.

34. Deacon, G. B.; Philips, R. J. Coord. Chem. Rev. 1980, 33, 227.

35. Ford, B. F. E.; Liengme, B. V.; Sams, J. R. J. Organomet. Chen. $1969,19,53$

36. Xie, Q.; Yang, Z. Q.; Zhang, Z. X.; Zhang, D. K. Appl. Organonet. Chem, 1992, 6, 193 .

37. Kalinowski, H. O.; Berger, S.; Brown, S. ${ }^{13}$ C NMR Spectroskopic; Thieme Verlag: Stuttgart, Germany, 1984.

38. Danish, M.; Ali, S.; Mazhar, M.; Badshah, A.; Tieknik, E. R. T. Moin Group Met. Chem. 1995, 18,697.

39. Parvez, M.; Ali, S.; Masood, T. M.; Mazhar, M.; Danish, M. Acta Cryst, 1997, C5, 1211 .

40. Meyer, B. N.; Ferrigni, N. R.; Pntnam, J. E.; Jacobson, L. B.; Nicholas, D. E.; McLaughlin, J. L.; Brine Shrimp, J. L. Planta Medica 1982, 45,31 .

4I. Krigman, M. R.; Silverman, A. P. Neurotoxicolog $1984,5,129$.

42. Barnes, J. M.; Stoner, H. B. Brit. J. Ind. Med. 1958, J5, 15.

43. Kazmi, \$. U.; Ali, \$. N.; Jamal, \$. A.; Rehman, A. J. Phar. Sci. 1991, 4, 113.

44. (a) Blank, H.; Rewbell, G Arch Derm. 1965, 92, 319. (b) Shaukat, S. S.; Khan, N. A.; Ahmed, F. Pak. J. Bot. 1980, J2,97.

45. Molloy, K. C. In The Chenistry of Metat-Carbon Bond; Hrtly, F. R. Ed.; Wiley: New York, 1989. 